\title{
A minimal representation of the self-assembly of virus capsids
}

\author{
J.M. Gomez Llorente J. Hernández-Rojas \\ Departamento de Física Fundamental II and IUdEA, Universidad de La Laguna, 38205, La Laguna, Tenerife, Spain
}

\begin{abstract}
Viruses are biological nanosystems with a capsid of protein-made capsomer units that encloses and protects the genetic material responsible for their replication. Here we show how the geometrical constraints of the capsomer-capsomer interaction in icosahedral capsids fix the form of the shortest and universal truncated multipolar expansion of the two-body interaction between capsomers. The structures of many of the icosahedral and related virus capsids are located as single lowest energy states of this potential energy surface. Our approach unveils relevant features of the natural design of the capsids and can be of interest in fields of nanoscience and nanotechnology where similar hollow convex structures are relevant.
\end{abstract}

\section{INTRODUCTION}

Viruses are among the simplest biological nano-systems, yet complex already at the molecular scale. All viruses have a capsid, i.e. a viral protein shell, that encloses the genetic material (RNA or DNA). It is formed by subunits called capsomers, which are composed of a variable number of proteins (from one to six). Despite their diversity, the coat proteins of many viruses spontaneously self-assemble in vivo and in vitro into the final viral capsid architecture.

About half of all viruses found so far have capsids with icosahedral symmetry ( $I$ symmetry group $)$. In many of these cases, there are 12 capsomers, called pentons, surrounded by 5 neighbour capsomers (i.e. with coordination number $n=5$ ); the other capsomers are called hexons and they have 6 neighbour capsomers $(n=6)$. The simple geometrical construction model introduced by Caspar and Klug $(\mathrm{CK})^{2}$ to explain the architecture of icosahedral viruses is a milestone in modern virology. Generalizations of the CK rules properly account for the geometry of some exceptional icosahedral capsids ${ }^{3-6}$ and other elongated virus capsids that share coordination numbers with the icosahedral ones ${ }^{7-9}$. Recent work ${ }^{10-12}$ provides important further development about the effect of the geometrical and topological constraints on the capsid structure.

The knowledge of the capsid interaction potential energy surface (PES) is a determining step in order to provide a correct description not only of the self-assembly process but also of the physical properties of the capsids. Apart from the computationally involved and physically hardly rewarding fullatom approach ${ }^{13}$, the goal of most of the work done so far on this matter is a good coarse-grained model of such PES. The simplest models represent the capsomers as isotropic bodies, but they require additional geometrical constraints such as a template of the virus capsid ${ }^{14}-19$. In other more complex models each capsomer is represented as a discrete set of either isotropic $\stackrel{17,20-24}{2}$ or anisotropic interaction centres $\stackrel{25-27}{=}$, or as a continuous body of interaction points plus some extra discrete centres $^{28}$.

Many complex systems often organize themselves following rather simple rules that emerge out of such complexity. The knowledge gathered about icosahedral virus capsids points towards this kind of simple organization in these systems. In the present work further evidence of this behaviour is revealed by finding the simplest form of a two-body interaction, when this is written as an expansion in the com- plete basis set of the angular terms of the standard multipolar expansions ${ }^{29}$, that incorporates the most relevant geometrical features of the real capsomer-capsomer interaction. This form will turn out to be universal and will unveil the important physical factors that determine the energetic stability of the capsid and its relationship with its geometrical structure. These factors must somehow be encoded in the virus genetic material and can become the target of biological evolution and of drug design.

\section{THE INTERACTION MODEL}

Our minimal model represents the capsomer $i$ as an unitary vector $\boldsymbol{v}_{i}$ at the position vector $\boldsymbol{r}_{i}$ in 3D space. The capsomer plane is supposed to be perpendicular to $\boldsymbol{v}_{i}$. Since, excluding pentons, the coordination number for the capsomers in icosahedral capsids is $n=6$ and this is the natural coordination for 2D isotropic interacting bodies, we will assume capsomers that are isotropic against rotations around $\boldsymbol{v}_{i}$. The orientational dependence of the interaction between two capsomers $(i, j)$ with relative position vector $\boldsymbol{r}_{i j}=\boldsymbol{r}_{j}-\boldsymbol{r}_{i}$ along $\boldsymbol{n}_{i j}=$ $\boldsymbol{r}_{i j} / r_{i j}$ will be expanded as a linear combination of the simplest multipolar terms. Namely, apart from the isotropic term (monopole-monopole), we will require the monopole-dipole terms $\boldsymbol{v}_{i} \cdot \boldsymbol{n}_{i j}$ and $\boldsymbol{v}_{j} \cdot \boldsymbol{n}_{i j}$, the two dipole-dipole terms $\boldsymbol{v}_{i} \cdot \boldsymbol{v}_{j}$ and $\left(\boldsymbol{v}_{i} \cdot \boldsymbol{n}_{i j}\right)\left(\boldsymbol{v}_{j} \cdot \boldsymbol{n}_{i j}\right)$, and the monopole-quadrupole contributions $\left(\boldsymbol{v}_{i} \cdot \boldsymbol{n}_{i j}\right)^{2}$ and $\left(\boldsymbol{v}_{j} \cdot \boldsymbol{n}_{i j}\right)^{2}$. If the $z$-axis of a body-fixed reference frame is chosen along $\boldsymbol{n}_{i j}$, then $\boldsymbol{v}_{i} \cdot \boldsymbol{n}_{i j}=\cos \theta_{i}$, $\boldsymbol{v}_{j} \cdot \boldsymbol{n}_{i j}=\cos \theta_{j}$, and $\boldsymbol{v}_{i} \cdot \boldsymbol{v}_{j}=\sin \theta_{i} \sin \theta_{j} \cos \left(\phi_{i}-\phi_{j}\right)+$ $\cos \theta_{i} \cos \theta_{j}$, where $\left(\theta_{i}, \phi_{i}\right)$ and $\left(\theta_{j}, \phi_{j}\right)$ are the polar coordinates of $\boldsymbol{v}_{i}$ and $\boldsymbol{v}_{j}$, respectively, in the body-fixed frame.

If the contact between two of our ideal capsomers occurs at the boundary of the capsomer planes, its equilibrium geometry will have a two-fold symmetry axis bisecting the dihedral angle between the planes. Therefore one must have $\phi_{i}-\phi_{j}=0$ and $\theta_{i}+\theta_{j}=\pi$. One can readily prove that the the form $-\boldsymbol{v}_{i} \cdot \boldsymbol{v}_{j}+\beta\left(\boldsymbol{v}_{i} \cdot \boldsymbol{n}_{i j}\right)\left(\boldsymbol{v}_{j} \cdot \boldsymbol{n}_{i j}\right)$ (rotationally invariant around the intercapsomer axis) with $\beta=2$ provides the unique and universal dipole-dipole interaction compatible with the geometrical constraints (the term is quite different from the electrostatic one which changes sign and has $\beta=3$ ). This ansatz does not depend on the dihedral angle $\Theta=\theta_{i}-\theta_{j}$.

It is well known that the value of the dihedral angle $\Theta$ is intimately connected to protein structure and strongly affects the 
capsid geometry. One can prove that the simplest combination of multipolar terms required to fix $\Theta$ must include monopoledipole and monopole-quadrupole terms in the unique form $\left(\boldsymbol{v}_{i} \cdot \boldsymbol{n}_{i j}\right)^{2}+\left(\boldsymbol{v}_{j} \cdot \boldsymbol{n}_{i j}\right)^{2}+2 \cos (\pi / 2-\Theta / 2)\left(\boldsymbol{v}_{i} \cdot \boldsymbol{n}_{i j}-\boldsymbol{v}_{j} \cdot \boldsymbol{n}_{i j}\right)$.

Remarkably, as will be shown later, no other angular terms will be generally required. A very convenient form of the capsomer-capsomer interaction that includes the isotropic and the previously fixed angular terms reads

$$
\begin{aligned}
& V_{i j}=4 \varepsilon_{i j}\left\{\left(\frac{\sigma_{i j}}{r_{i j}}\right)^{2 m}-\left(\frac{\sigma_{i j}}{r_{i j}}\right)^{m}\right. \\
\times & {\left[1+\alpha_{i j}\left[\boldsymbol{v}_{i} \cdot \boldsymbol{v}_{j}-\beta_{i j}\left(\boldsymbol{v}_{i} \cdot \boldsymbol{n}_{i j}\right)\left(\boldsymbol{v}_{j} \cdot \boldsymbol{n}_{i j}\right)-\zeta_{i j}\right]\right.} \\
- & \left.\left.\frac{1}{2} \gamma_{i j}\left[\left(\boldsymbol{v}_{i} \cdot \boldsymbol{n}_{i j}+\eta_{i j}\right)^{2}+\left(\boldsymbol{v}_{j} \cdot \boldsymbol{n}_{i j}-\eta_{i j}\right)^{2}\right]\right]\right\},
\end{aligned}
$$

where $m$ is an integer power and all greek letters are the other potential parameters. We know that $\beta_{i j}$ is a critical parameter and the calculations show that its value can not deviate too much from its theoretical value of 2 . Somewhat less critical is $\eta_{i j}=\cos (\pi / 2-\Theta / 2)$, which fixes the dihedral angle $\Theta$ when $\gamma_{i j} \neq 0$. Besides $\alpha_{i j}, \gamma_{i j}, \zeta_{i j} \geq 0$ is required, the results being quite robust in wide intervals of their values. We have set $\alpha_{i j}=1 . \zeta_{i j}$ is a positive shift parameter that affects the intercapsomer equilibrium distance; for $\beta_{i j}=2$ its most convenient value is $\zeta_{i j}=1$ and, in this case, the binding energy of the capsomer dimer is the same independent of the values chosen for the parameters $\alpha_{i j}, \gamma_{i j}$ and $\eta_{i j}$, and its equilibrium distance is always $r_{i j}^{\mathrm{e}}=2^{\frac{1}{m}} \sigma_{i j}$. The value of the power $m$ in Eq. 1 is not of critical importance either ${ }^{30}$. We have chosen for most calculations $m=12$. The total interaction energy for a set of $N$ capsomers will be evaluated as the sum of all pair interactions, namely $V=\frac{1}{2} \sum_{i \neq j}^{N} V_{i j}$.

We have performed calculations with one type (hexons) and two types (pentons and hexons) of capsomers. For the hexonhexon potential we have set $\varepsilon_{i j}=\varepsilon_{\mathrm{hh}}=1$ as the energy unit and $\sigma_{i j}=\sigma_{\mathrm{hh}}=1$ as the length unit. In general, we have set $\gamma_{i j}=\gamma_{\mathrm{hh}}=0$, thus the hexon-hexon dihedral angle is left free so that it can adapt to non equivalent geometrical environments without frustration. For capsids with only hexons, a non-zero value of $\gamma_{i j}$ may be required to favour the formation of a given capsid structure, and in such cases we have chosen $\gamma_{\mathrm{hh}}=5$. The penton-penton potential parameters are not critical; our choice for them was $\varepsilon_{i j}=\varepsilon_{\mathrm{pp}}=0.1, \sigma_{i j}=\sigma_{\mathrm{pp}}=1$ and $\gamma_{i j}=\gamma_{\mathrm{pp}}=0$.

When pentons are present, each penton should have five hexon neighbours. These local structures are critical in the self-assembly of hollow capsids and we have fixed the parameters of the hexon-penton pair potential to favour their formation; namely we have chosen $\varepsilon_{i j}=\varepsilon_{\mathrm{hp}}=$ 1.5 and $\sigma_{i j}=\sigma_{\mathrm{hp}}=2^{\frac{m-1}{m}} R \sin \vartheta_{\mathrm{hp}}$, with $\vartheta_{\mathrm{hp}}=$ $\frac{1}{2} \arcsin \left[\sigma_{\mathrm{hh}} /\left(2^{\frac{m-1}{m}} R \sin \frac{\pi}{5}\right)\right]$; with the latter value for $\sigma_{\mathrm{hp}}$ the pentagonal pyramid formed by a central penton surrounded by five hexons located at the vertices of a regular hexagon whose edge lengths are the corresponding capsomercapsomer equilibrium distances will have all its vertices lying on the surface of a sphere whose radius is $R$. Although not necessary in most of the capsid structures found, surface curvature can be further fixed through the hexon-penton interaction by choosing a non vanishing $\gamma_{i j}$. We have generally chosen $\gamma_{i j}=\gamma_{\mathrm{hp}}=5$ and $\eta_{i j}=\eta_{\mathrm{hp}}=2^{\frac{1-m}{m}} \sigma_{\mathrm{hp}} / R$; this last choice induces a curvature radius $R$ for an hexon-penton pair at its equilibrium distance. The same value was also used for $\eta_{\mathrm{hh}}$ in capsids made only of hexons.

The only free parameter in our potential model is therefore $R$. Obviously $R$ should depend on the total number of capsomers, $N$, in the capsid. We have varied it with the simple relation $R=2^{1 / m} \sigma_{\mathrm{hh}}\left[\frac{\sqrt{3}(N-2)}{8 \pi}\right]^{1 / 2}$, which is obtained from the surface of the unfolded capsid icosahedron on the $2 \mathrm{D}$ hexagonal lattice.

\section{RESULTS AND DISCUSSION}

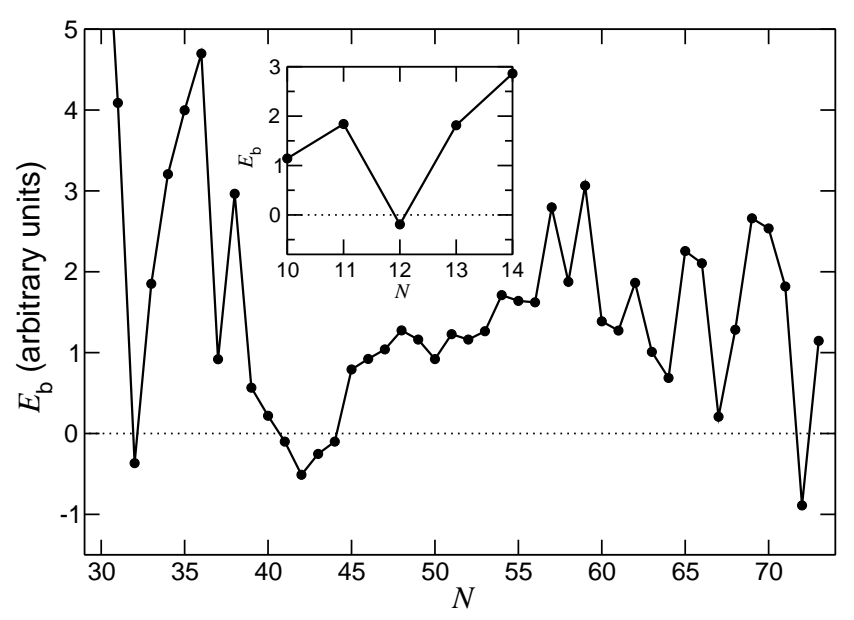

FIG. 1: Capsid binding energies $E_{\mathrm{b}}$ as a function of the capsomer number $N$. For the main curve the number of pentons is fixed to 12 . For the inset figure all capsomers are of the same type (hexons in our notation).

We will name the different capsids, when possible, with their corresponding triangulation numbers $T^{2.31}, Q_{5} \mathrm{~F}^{\frac{7-9}{2}}$, $Q_{3 \mathrm{~F}}, Q_{2 \mathrm{~F}}{ }^{\frac{9}{2}}$. Basin-hopping global optimization ${ }^{32-34}$ was used to find the lowest energy structures. We have defined conveniently shifted binding energies $E_{\mathrm{b}}=V+(3 N-6) \varepsilon_{\mathrm{hh}}+$ $5 N_{\mathrm{p}}\left(\varepsilon_{\mathrm{hp}}-\varepsilon_{\mathrm{hh}}\right), N_{\mathrm{p}}$ being the number of pentons. With this choice a capsid with optimal packing and contact interactions would have $E_{\mathrm{b}}=0$. Values of $E_{\mathrm{b}}$ slightly lower than zero are possible due to the (small) long-range tail of our interaction. $E_{\mathrm{b}}$ is also a natural and convenient measure of frustration for the class of viral capsids considered in this work; namely, capsids with $E_{\mathrm{b}} \lesssim 0.5$ present low frustration structures, i.e. structures with optimal coordination for each capsomer and pair interaction energies $V_{i j}$ between neighbour capsomers close to their minimum values.

The calculations performed using only one type of capsomers (i.e. hexons) and the simplest form of the interaction with free dihedral angle (i.e. $\gamma_{\mathrm{hh}}=0$ ) provide hollow lowestenergy structures ${ }^{30}$. Particularly stable capsids are those with 
icosahedral symmetry corresponding to the allowed triangulation numbers $T$. There are indeed real capsids made of only one type of capsomers ${ }^{30}$ and some of our forms have been reproduced with other theoretical models $15,17,18,28,35$. However in this case competing structures appear close in energy to the global minima, this behaviour being a consequence of the relatively large frustration of those minima, with energies $E_{\mathrm{b}} \gtrsim 10$ that increase with $N$. Such competition can be reduced significantly for icosahedral capsids by choosing $\gamma_{\mathrm{hh}} \neq 0$ and an adequate dihedral angle. Nevertheless, this procedure never reduces frustration. The only way to do so is by replacing the hexons with coordination $n=5$ by pentons with the right structure. Thus 12 pentons are required in agreement with Nature's choice for most of the icosahedral an related virus capsids. Henceforth we will focus our attention on these capsids made of hexons and 12 pentons.

Figure $\mathrm{S}-4$ presents the global minimum energies $\left(E_{\mathrm{b}}\right)$ as a function of the capsomer number $N$. A relevant property of our model is that if the curvature parameter $R$ is included in the global optimization procedure, its stabilizing effect is significantly more important for the icosahedral structures. Capsids corresponding to the allowed triangulation numbers



$T=1$

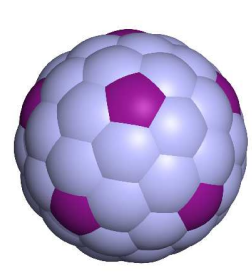

$T=7$

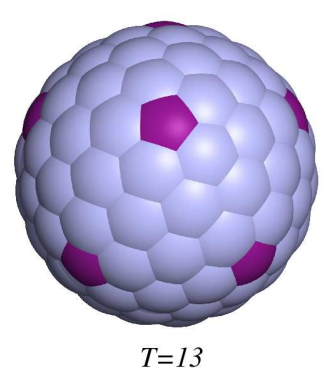

FIG. 2: The structure of icosahedral capsids found as global energy minima in our PES. Capsomers with coordination number $n=5$ are purple. With our parameter choice, these are hexons for the $T=1$ capsid and pentons for all other capsids. Light-blue capsomers are all hexons.

$T$ up to $T=16$ (some of these outside the range in Figure [S-4], have the icosahedral structures as the lowest energy and optimal packing structures, having binding energies closer to or smaller than zero and also lower than those of their neighbours in capsomer number. Illustrations of these icosahedral capsids are shown in Figure 2. In the $T$ range studied, we found a relevant exception to the previous rule for $N=42$, which would correspond to a capsid with $T=4$. The lowest energy structure found in this case corresponds to a non icosahedral spheroidal geometry given by the two triangulation numbers $T=4, Q_{5 \mathrm{~F}}=2$, and having the symmetry of the $D_{5}$ point group (see Figure $3 \mathrm{~b}$ ). The corresponding $T=4$ capsid with icosahedral geometry has an energy only 0.01 units above the global energy minimum. Both capsids have practically the same optimal $R$, and therefore no discrimination between them is possible by changing the curvature. This result points to one of two possible consequences: either the virus capsid could adopt both structures without functional implications, or additional terms would be required in our interaction model to include geometrical constraints that would eliminate one of the competing geometries without adding significant frustration to the other. The higher symmetry of the icosahedral capsid makes much easier the discrimination in favour of this structure. Namely, we have been able to remove the competition of the $D_{5}$ capsid by adding some terms to the penton-hexon and hexon-hexon interactions that incorporate the $C_{2}$ symmetry of hexons in the icosahedral capsid (details will be presented elsewhere). One can easily find that similar non icosahedral but spheroidal structures are possible for all $T$ numbers that do not coincide with the corresponding class number $P^{30}$.

Another optimal packing structure corresponding to $N=$ 42 is that of the $\Phi 29$ virus capsid. This has a prolate geometry with $T=3$ and $Q_{5 \mathrm{~F}}=5$ 年. Our model is also able to provide this structure (Figure 3k), which can be made the lowest energy structure by optimizing its curvature $R$ after conveniently increasing the value of the $\gamma_{\mathrm{hp}}$ parameter.

For capsomer numbers $N$ between the values corresponding to the allowed triangulation numbers $T$, we find capsids with optimal packing but higher frustration. Examples are included in Figure 3 Some of them follow Moody's.8 (oblate for $N=67$ in Figure $3 \mathrm{~h}$ ) and Luque and Reguera's $\mathrm{s}^{9}$ rules ( $N=46$ and 48 in Figure 3 e and f, respectively), but others are completely new $(N=40$ with tetrahedral symmetry in Figure $3 \mathrm{a}, N=63$ with $D_{3}$ symmetry in Figure $3 \mathrm{~g}$, or the asymmetric $N=45$ in Figure 3 d). Figure $\mathbf{S}-4$ shows particularly stable capsids for $N$ in the neighbourhood of the $T=4$ capsid (e.g. $N=41,43,44)$ that have spheroidal geometries of the new types with some remaining symmetry (e.g. $D_{3}$, $C_{2}, S_{4}$, respectively).

The larger capsid that we have been able to find is that of the prolate bacteriophage $\mathrm{T} 4$ head. This has a capsomer number $N=167$, with $T=13$ and $Q_{5 \mathrm{~F}}=20^{37}$. Two views of this capsid are shown in Figure 3 (i and j). After optimizing the curvature $R$, the energy for this capsid is $E_{\mathrm{b}}=5.97$, which indicates an important frustration. Similar behaviour for the frustration is also found in the larger icosahedral capsids $T \geq 9$, in agreement with geometrical considerations 11 . By slightly modifying our potential mode $\mathrm{l}^{30}$, we have been able to incorporate in it the known buckling process ${ }^{38}$ and 




FIG. 3: The structure of non-icosahedral capsids found as global energy minima in our PES. Purple capsomers are pentons while lightblue ones are hexons.

reduce significantly the frustration. The illustrations for this capsid in Figure 3 include this process, which is particularly evident in its top view.

In order to explore the available capsid configuration space as a function of temperature $T^{*}$, we have used parallel tempering Monte Carlo sampling ${ }^{39-41}$ of such space for the $T=3$ capsid $^{30}$. First of all, let us point out that since our model assumes unstructured capsomers, it can not account for processes known to affect the structure of such units ${ }^{42}$. However this sampling provides relevant information about possible capsid structures before the disassembly transition. The ideal caloric curves obtained from this statistical analysis 30 show the capsid disassembly as a first-order-like phase transition with a sharp change in the internal energy. Moreover, at temperatures below this transition, we only find vibrationally excited forms of the optimal capsid structure.

\section{CONCLUSIONS}

By taking into account geometrical constraints, we have derived the shortest and yet quite universal form of a truncated multipolar expansion for the capsomer-capsomer pair interaction in icosahedral capsids. For capsids made of only one kind of capsomers a simple form without adjustable parameters provides a great variety of hollow structures. The simplicity and universality of this form makes it a promising tool in the understanding of the physical properties of homogeneous hollow structures of relevance in many applied fields of nanoscience and nanotechnology (membranes, colloidal surfaces, etc.). The introduction of a different type of capsomers (12 pentons) eliminates competing structures and reduces frustration significantly. In this case, the curvature $R$ is the only relevant parameter of the model. By varying this parameter and the number of capsomers $N$, the model is able to provide many optimal packing structures as global energy minima. Apart from the allowed icosahedral structures, we find geometries satisfying Moody's, 7,8 and Luque and Reguera's rules ${ }^{9}$, and other completely new structures. This simple form unveils the following relevant physical features of the natural design of the viral capsids.

The stabilizing effect of an optimal choice of the curvature is significantly larger for the icosahedral capsids. Besides, prolate structures (e.g. $\Phi 29$ virus) can be stabilized in this way against competing icosahedral capsids (e.g. $T=4$ ).

After curvature optimization (the $T=4$ capsid requiring also additional interaction terms), self-assembly is dominated and guided by a single capsid structure.

For the larger capsids ( $T \geq 9$ or $N>90$ ) we observe an increase of frustration even after curvature optimization. Buckling has been shown to reduce such frustration.

There is still a last conclusion of fundamental relevance. This concerns the physical reasons behind the simplicity yet general validity of our interaction model. Of course, the highest possible symmetry of icosahedral capsids is a first main reason; the second one is the $2 \mathrm{D}$ close-packing arrangement of the capsomers, which all except the pentons have coordination number $n=6$. As a matter of fact this packing is the easiest one to reproduce by pair interaction models. These two reasons are related by the fact that of all finite rotation point groups, $I$ is the one that has more symmetry operations in correspondence with those of the close-packing 2D hexagonal lattice.

Another important physical reason behind simplicity is in the principle of low frustration satisfied by many-body systems with a unique lowest energy structure, as should occur, for obvious reasons, in the case of viral capsids. In an appropriate expansion of the system potential interaction energy (as the multipolar one used here), the principle of low frustration seems to reduce the number of terms required to reproduce the optimal structure. Of course, many terms will have to be added to our universal form to approach a particular real interaction, but these should hardly affect both the capsid structure and its binding energy. They will certainly change the statistical weight of the configuration space around that structure and perhaps eliminate other competing geometries, as we have shown for the $T=4$ capsid. 


\section{Acknowledgement}

We thank Prof. David Reguera and Prof. David J. Wales for their valuable comments on this work.

* Electronic address: jmgomez@ull.es

$\dagger$ Electronic address: jhrojas@ull.es

$\ddagger$ Electronic address: jbreton@ull.es

1 A. Zlotnick, J. Mol. Recognit., 2005, 18, 479-490.

2 D. L. D. Caspar and A. Klug, Cold Spring Harbor Symp. Quant. Biol., 1962, 27, 1-24.

${ }^{3}$ R. A. Twarock, J. Theor. Biol., 2004, 226, 477-482.

4 T. Keef, C. Micheletti and R. Twarock, J. Theor. Biol., 2006, 242, 713-721.

${ }^{5}$ V. L. Lorman and S. B. Rochal, Phys. Rev. Lett., 2007, 98, 185502.

${ }^{6}$ V. L. Lorman and S. B. Rochal, Phys. Rev. B., 2008, 77, 224109.

${ }^{7}$ M. F. Moody, Virology, 1965, 26, 567-576.

${ }^{8}$ M. F. Moody, J. Mol. Biol., 1999, 293, 401-433.

9 A. Luque and D. Reguera, Biophys. J., 2010, 98, 2993-3003.

${ }^{10}$ R. V. Mannige and C. L. Brooks, III, Phys. Rev. E, 2008, 77, 051902.

11 R. V. Mannige and C. L. Brooks, III, Proc. Natl. Acad. Sci. USA, 2009, 106, 8531-8536.

12 R. V. Mannige and C. L. Brooks, III, PLoS ONE, 2009, 5, e9423.

13 P. L. Freddolino, A. S. Arkhipov, S. B. Larson, A. McPherson and K. Schulten, Structure, 2006, 14, 437-449.

14 R. F. Bruinsma, W. M. Gelbart, D. Reguera, J. Rudnick and R. Zandi, Phys. Rev. Lett., 2003, 90, 248101.

15 R. Zandi, D. Reguera, R. F. Bruinsma, M. Gelbart and J. Rudnick, Proc. Natl. Acad. Sci. USA, 2004, 101, 15556-15560.

${ }^{16}$ R. Zandi and D. Reguera, Phys. Rev. E, 2005, 72, 021917.

17 T. Chen, Z. Zhang and S. C. Glotzer, Proc. Natl. Acad. Sci. USA, 2007, 104, 717-722.

18 T. Chen and S. C. Glotzer, Phys. Rev. E, 2007, 75, 051504.

19 A. Luque, R. Zandi and D. Reguera, Proc. Natl. Acad. Sci. USA, 2010, 107, 5323-5328.

20 D. C. Rapaport, Phys. Rev. E, 2004, 70, 051905.

21 D. J. Wales, Phil. Trans. Roy. Soc. A, 2005, 363, 357-377.

${ }^{22}$ H. D. Nguyen, V. S. Reddy and C. L. Brooks, III, Nano Lett., 2006, 7, 338-344.

${ }^{23}$ H. D. Nguyen and C. L. Brooks, III, Nano Lett., 2008, 8, 4574 4581.

${ }^{24}$ S. N. Fejer, T. R. James, J. Hernández-Rojas and D. J. Wales, Phys. Chem. Chem. Phys., 2009, 11, 2098-2104.

25 M. F. Hagan and D. Chandler, Biophys. J., 2006, 91, 42-54.

${ }^{26}$ K. V. Workum and J. F. Douglas, Phys. Rev. E, 2006, 73, 031502.

27 O. M. Elrad and M. F. Hagan, Nano Lett., 2008, 8, 3850-3857.

28 S. N. Fejer, D. Chakrabarti and D. Wales, ACSNano, 2010, 4, 219 228.

${ }^{29}$ L. C. Biedenharn and J. D. Louck, Angular Momentum in Quantum Physics: Theory and Application, Cambridge University Press, 1984.

30 See the Electronic Supplementary Information (ESI).

31 D. L. D. Caspar, Biophys. J., 1980, 32, 103-138.

32 D. J. Wales and J. P. K. Doye, J. Phys. Chem. A, 1997, 101, 51115116.

33 Z. Q. Li and H. A. Scheraga, Proc. Natl. Acad. Sci. USA, 1987, 84, 6611-6615.

${ }^{34}$ D. J. Wales, Energy Landscapes, Cambridge University Press, 2003.
35 I. G. Johnston, A. A. Louis and J. P. K. Doye, J. Phys.: Condens. Matter, 2010, 22, 104101.

36 Y. Tao, N. H. Olson, W. Xu, D. L. Anderson, M. G. Rossmann and T. S. Baker, Cell, 1998, 95, 431-437.

37 A. Fokine, P. R. Chipman, P. G. Leiman, V. V. Mesyanzhinov, V. B. Rao and M. G. Rossmann, Proc. Natl. Acad. Sci. USA, 2004, 101, 6003-6008.

${ }^{38}$ W. R. Wikoff, J. F. Conway, J. Tang, K. K. Lee, L. Gan, N. Cheng, R. L. Duda, R. W. Hendrix, A. C. Steven and J. E. Johnson, J. Struct. Biol., 2006, 153, 300-306.

39 C. J. Geyer, in Computing Science and Statistics: Proceedings of the 23rd Symposium on the Interface: 1991, ed. E. M. Keramidas, Interface Foundation, Fairfax Station, VA, 1991, p. 156.

40 D. J. Earl and M. W. Deem, Phys. Chem. Chem. Phys., 2005, 7, 3910-3916.

41 J. P. Neirotti, F. Calvo, D. L. Freeman and J. D. Doll, J. Chem. Phys., 2000, 112, 10350-10357.

${ }^{42}$ S. F. Ausar, T. R. Foubert, M. H. Hudson, T. S. Vedvick and C. R. Middaugh, J. Biol. Chem., 2006, 281, 19478-19488. 


\section{SUPPLEMENTARY MATERIAL}

In the Article we use, when possible, the notation introduced by Caspar and Klug to label the icosahedral capsids. These authors build a geometrical model of the capsid by folding a 2D hexagonal lattice whose equivalent positions are given by a pair of non negative integers $(h, k)$. Then the allowed structures are those having the triangulation number $T=h^{2}+k^{2}+h k$, (whose possible values are $T=1,3,4,7,9,12,13,$. ). The number $T$ can be written as $T=P f^{2}$, where $f$ is the greatest common divisor of $h$ and $k$, and each allowed $P$ value corresponds to a capsid class. With this construction the number of hexons is $10(T-1)$ and one always has 12 pentons. Some other structures have been denoted with one additional triangulation number $Q_{5 \mathrm{~F}}$ (see References 7-9 in the Article), $Q_{3 \mathrm{~F}}$ or $Q_{2 \mathrm{~F}}$ (see Ref. 9 in the Article).

An important mathematical result has guided us in the construction of our capsomer-capsomer interaction model: the anisotropic terms (those depending on the angular orientation) of the standard multipolar expansion of the interaction between two charge distributions form a complete basis set to expand the angular dependence of the interaction between any two bodies. This basis set is the direct product of two Wigner matrix basis sets (Ref. 29 in the Article), one for each of the two bodies.

Notice that in our interaction energy expression $\boldsymbol{v}_{i}$ is not an electric dipole, but a vector giving the capsomer orientation. Of course, the origin of these interaction terms has to be found in the inter-residue forces existing between the proteins forming each capsomer. These forces have not only electrostatic contributions, but also dispersive, inductive and thermodynamical (through the water solvent) ones.

The parameter $m$ in our model (Eq. 1 in the Article) determines somehow the potential range relative to the capsomer size. Interactions between real viral capsomers are thought to be very short-ranged, requiring values of this parameter as high as $m \sim 50$. We have chosen for most calculations a lower value $(m=12)$ for practical computational reasons.

Notice that if in the expression giving the interaction energy (Eq. 1 in the Article) $\alpha_{i j}$ and $\gamma_{i j}$ are set to zero and $m=6$, then $V_{i j}$ takes the form of the Lennard-Jones potential with $\varepsilon_{i j}$ being the depth of the interaction well and $\sigma_{i j}$ the finite distance below which $V_{i j}>0$.

Our results showed that frustration is significantly important for the larger capsids, e.g. the bacteriophage $\mathrm{T} 4$ head and the icosahedral capsids with $T \geq 9$. We also mentioned that by modifying our interaction model to allow for the buckling process such frustration could be reduced. Let us explain now this modification. The faceting induced by buckling in the capsid affects particularly to the hexons at the edges of the icosahedron. These have to bend somehow to adapt to the orientation of the two corresponding faces. These bending degrees of freedom have been introduced by adding two new unitary vectors $\left\{\boldsymbol{v}_{i k}, k=1,2\right\}$ for each hexon, being their orientation the 4 new degrees of freedom (more details will be given elsewhere). Then, the penton-hexon interaction was left unchanged using the original $\boldsymbol{v}_{i}$, and the anisotropic part, $V_{i j}^{(\mathrm{a})}$, of the hexon-hexon interaction (remember that $\gamma_{\mathrm{hh}}=$ 0 ) was changed to

$$
\begin{gathered}
V_{i j}^{(\mathrm{a})}=-4 \varepsilon_{\mathrm{hh}}\left(\frac{\sigma_{\mathrm{hh}}}{r_{i j}}\right)^{m}\left\{\operatorname { m a x } _ { k , l = 1 , 2 } \left\{1+\alpha_{\mathrm{hh}}\right.\right. \\
\left.\times\left[\boldsymbol{v}_{i k} \cdot \boldsymbol{v}_{j l}-\beta_{\mathrm{hh}}\left(\boldsymbol{v}_{i k} \cdot \boldsymbol{n}_{i j}\right)\left(\boldsymbol{v}_{j l} \cdot \boldsymbol{n}_{i j}\right)-\zeta_{\mathrm{hh}}\right]\right\} \\
\left.+\frac{1}{2} \kappa_{\mathrm{hh}} \sum_{k=1}^{2}\left[\left(\boldsymbol{v}_{i}-\boldsymbol{v}_{i k}\right)^{2}+\left(\boldsymbol{v}_{j}-\boldsymbol{v}_{j k}\right)^{2}\right]\right\},
\end{gathered}
$$

where max stands for the maximum value of all $(k, l)$ pairs. A penalty energy for this bending has been added with the term proportional to $\kappa_{\mathrm{hh}}$. The penalty parameter is $\kappa_{\mathrm{hh}}>0$, and for $\kappa_{\mathrm{hh}} \rightarrow \infty$ this expression reduces to the form included in Eq. 1 of our Article.

Let us comment now on the physical meaning of the arbitrary energy unit used in our model. From the knowledge of the interaction between capsomers at the molecular level (S. Reddy, H. A. Giesing, R. T. Morton, A. Kumar, C. B. Post, C. L. Brooks, III and J. E. Johnson, Biophys. J., 1998, 74, 546-558), an estimate of the actual value of this energy unit is $\varepsilon=1 \sim 10 k_{\mathrm{B}} T_{\circ}^{*}$ units or even higher $\left(k_{\mathrm{B}}\right.$ is the Boltzmann constant and $T_{\circ}^{*}$ the normal temperature).
This would imply, for instance, that any other capsid structure differing from

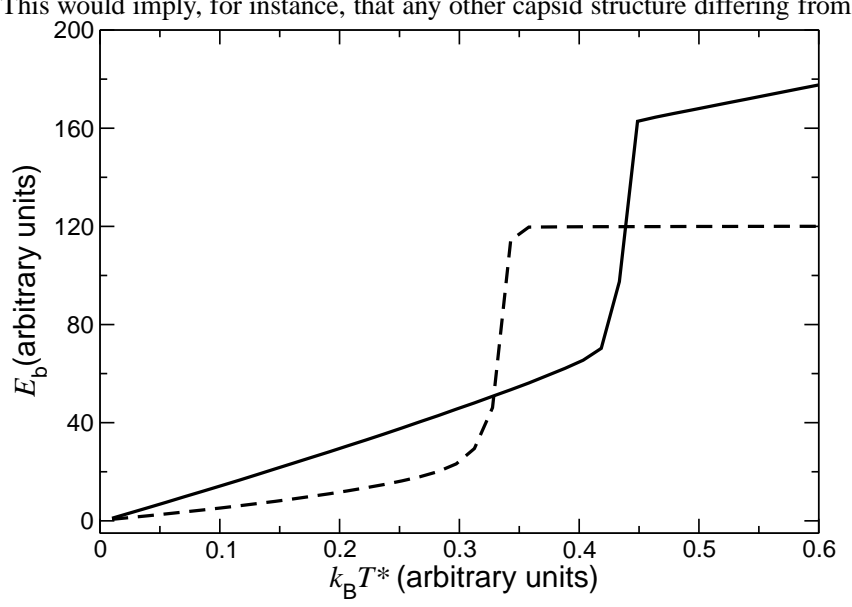

FIG. S-4: Caloric curve giving the average internal energy $E_{b}$ as a function of temperature $T^{*}$ ( $k_{\mathrm{B}}$ is the Boltzmann constant) for the icosahedral capsid with triangulation number $T=3(N=32)$. The continuous line was obtained with a value of the range parameter $m=12$ (Eq. 1 in the Article), while the dashed line is for $m=48$.

the lowest energy one in just $\sim 0.2$ of our energy units would be statistically irrelevant at normal temperatures.

Except for capsids in the neighborhood of the $T=1(N=12)$ capsid, we have presented in the Article results obtained for a fixed number (12) of pentons and a changing number of hexons. However we have also performed more extensive calculations using only one type of capsomers. In this case we have used the parameters of the hexon-hexon interaction with vanishing and non-vanishing $\gamma_{\mathrm{hh}}$ values. In both cases we obtain hollow capsids, with a larger diversity of forms in the case $\gamma_{\mathrm{hh}}=0$. A sharper energetic discrimination between them requires a non-vanishing $\gamma_{\mathrm{hh}}$ and an adequate choice of the curvature parameter $R$. In this way we could be able to generate capsids sharing the structure of those obtained with the inclusion of the two types of capsomers. A particular example is the structure of the human papilloma virus capsid, which is known to be made of just one type of capsomers. Our model is able to also reproduce the structure of this particular capsid. Its theoretical triangulation number is $T=7(N=72)$. Besides our structure shows, as the experimental reconstruction (T. S. Baker, W. W. Newcomb, N. H. Olson, L. M. Cowsert, C. Olson and J. C. Brown, Biophys. J., 1991, 60, 1445-1456), some slight asymmetries. However, as discussed in the Article, this homogeneneous structure has a relativeley large frustration, with a bonding energy $E_{\mathrm{b}} \simeq 20$. By replacing the 12 hexons with coordination $n=5$ by pentons this energy can be reduced to a value $E_{\mathrm{b}} \simeq-1$.

The capsid likely lowest energy structures provided by our interaction potential model were located using the basin-hopping scheme (Ref. 32 in the Article), which is also known as the "Monte Carlo plus energy minimization" approach of Li and Scheraga (Ref. 33 in the Article). This method is very well known and has been used successfully in the fields of clusters, biomolecules and glasses (Ref. 34 in the Article).

Our parallel tempering Monte Carlo sampling calculations make use of standard methods (References 39-41 in the Article). Figure S-4 gives, with a continuous line, the ideal caloric curve for the $T=3$ icosahedral capsid obtained with the potential-range parameter $m=12$. The ideal transition temperature is $k_{\mathrm{B}} T^{*}=0.43$ in this case. We have argued that from the relative length scales of the capsomer size and the interaction range values as high as $m \sim 50$ would be required. While the particular choice of this parameter hardly affects the structures of the global energy minima (we have tested choices in the range $6<m<48$ which provide rather similar structural results), it has significant effects on the theoretical disassembly transition. Namely for $m=48$ this ideal transition temperature is reduced to $k_{\mathrm{B}} T^{*}=0.32$ (dashed line in Figure $\mathrm{S}-4$. 